\section{P-174 HOSPICE GP TRAINEE INDUCTION - BETTER PRESCRIBING, FEWER INCIDENTS}

Ros Marvin, Sarah Bell. Garden House Hospice Care, Letchworth Garden City, UK

10.1136/spcare-2021-Hospice.190

An increase in hospice clinical incidents was identified during the pandemic in 2020. Investigation of medical team incidents demonstrated the requirement for additional education of new GP trainees on four-monthly hospice placements, specifically targeting prescribing. GP trainees learn key palliative medicine and hospice processes in a single day of induction. Previously, induction focussed on medical care, with the processes, including prescribing, largely learnt 'on the job'. Analysis of the incidents identified key areas for increased education: lack of experience at prescribing anticipatory medications; errors due to using an unfamiliar prescription chart; and the complex process of ordering discharge medications from the local hospital pharmacy.

A new, interactive prescribing practice induction session was created: prescribing anticipatory medications; error identification on hospice prescription charts; and discharge prescribing on TTO forms. This was delivered to two consecutive groups of new GP trainees. Contemporaneous feedback on prescribing was given to trainees during the session.

Numerical comparison of medical team clinical incidents demonstrated 21 incidents in the 12 months prior to the new induction (average 1.75 incidents per month), versus six incidents in the six months post new induction (average 1 per month), a reduction of over $40 \%$.

Feedback from trainees regarding the induction session has been universally positive (8/8 trainees), with one trainee commenting 'excellent... detailed training in using the system and ... medications [for patients] to take home'. The resulting reduction in incidents demonstrates improved patient care and safety.

\section{P-175 IS THE JRCPTB PALLIATIVE MEDICINE CURRICULUM FIT FOR PURPOSE?}

Michelle Hinchliffe. Airedale NHS Foundation Trust, Keighley, UK

10.1136/spcare-2021-Hospice.191

Why? The structure of medical training in the UK is currently undergoing a major review. The current plan is for trainees in palliative care to be dual accredited with internal medicine. However, this will mean extensive changes to the curriculum. It is of high importance to establish which aspects of the current palliative medicine curriculum are most valued and where there is scope for change.

How? A focus group discussion was carried out with five palliative medicine trainees, focusing on: Most important areas of the curriculum; Relevance; Anything missing?; Future development?; How this fits with Shape of Training.

Analysis of the transcript highlighted four major themes. These themes were then put to consultants for comment.

Results Four major themes emerged from the trainee focus group:

1. Expansion of non-clinical areas of the curriculum, in order to further develop management, leadership and supervisory skills.
2. Generalisation of the clinical components, to incorporate more general medicine and not make it so specific that trainees struggle to achieve assessments in each aspect.

3. A different approach to assessment was felt to be needed. In particular, not all DOPS were felt to be relevant and more scope for assessment in non-clinical areas was discussed.

4. Flexibility to focus on an area of interest within training, such as teaching, research or a particular clinical interest.

Key points from consultant responses

- Overall agreement with many points.

- The flexibility to focus on an area of interest within training may be difficult to achieve and actually may be more appropriate to focus on developing as a consultant.

Conclusion These findings provide a basis for ongoing exploration of trainers' and trainees' priorities for future development of the palliative medicine curriculum. It is important to consider the views of those interacting with the curriculum in their daily practice in order to develop a tool that can truly meet the needs of the specialty.

\section{P-176 EVALUATION OF HOSPICE BASED EXPERIENTIAL PLACEMENTS FOR FOUNDATION TRAINEE PHARMACISTS}

Amy Mundell. Newcastle upon Tyne NHS Foundation Trust, Newcastle upon Tyne, UK

10.1136/spcare-2021-Hospice. 192

Background To qualify as a pharmacist in the UK, a four-year Master of Pharmacy (MPharm) degree followed by a year of foundation training is completed. Experiential learning placements, where the trainees learn through reflection contribute to this training. Few universities offer experiential learning placements within hospices, and where they do, duration is very limited varying between $3-8$ hours per week. Such placements have not previously been reported in foundation training. There is considerable breadth and depth of opportunities for pharmacists' involvement in hospice care, specifically the ability to positively affect patient outcomes and become valued members of the multidisciplinary team.

Aim(s) Provision and evaluation of an experiential learning placement at St Oswald's Hospice, Newcastle for Foundation Trainee Pharmacists from Newcastle upon Tyne NHS Foundation Trust.

Methods The evaluation was a questionnaire performed both before and after the placement, based upon trainees' confidence in 16 aspects of patient care and medicines management pertinent to hospice pharmacy practice and based upon their governing body's learning outcomes.

Results Eight trainee pharmacists completed the placement and five performed the evaluation. Prior to the placement $60 \%$ of the trainees did not feel confident having discussions with patients with life-limiting illnesses reducing to $20 \%$ following placement completion. Only $40 \%$ of trainees understood the financial responsibilities of a pharmacist's role before the placement compared to $80 \%$ after. When asked about how confident the trainees felt ensuring safe prescriptions for patients with compromised organ function, only $20 \%$ agreed prior to the placement increasing to $60 \%$ after.

Conclusions The results show a logical trajectory prior to and following the Experiential-Learning-Trajectory. The 
understanding of hospice care is lacking in trainee pharmacists due to minimal hospice training. The Experiential-LearningTrajectory helped improve the trainees' confidence in understanding a pharmacists' contribution to hospice care. Further placements and evaluation using higher levels of Moore's Evaluation Framework is planned.

\section{P-177 EXPLORATION OF ATTENDANCE ACROSS 28 ECHO PROJECT ECHO NETWORKS IN THE CONTEXT OF EVERYDAY PRACTICE}

${ }^{1} J a n e t$ Diffin, ${ }^{1}$ Tracey McTernaghan, ${ }^{2}$ Aine McMullan, ${ }^{2}$ Martin Hayes, ${ }^{1}$ Max Watson, ${ }^{1}$ Christopher Jenkins. ${ }^{1}$ Hospice UK, Belfast, UK; ${ }^{2}$ Health and Social Care Board, Belfast, UK

\subsection{6/spcare-2021-Hospice.193}

Introduction Many studies that have included reports of attendance at ECHO sessions have focused on networks that have purposively selected participants to attend. This data may not provide an accurate reflection of participation rates within the constraints of everyday practice.

Aim The aim of this study was to explore attendance patterns across ECHO networks attended by both medical and social care professionals within the confines of normal working practice, and identify the reasons for any observed variations.

Methods Network activity data were collected from 28 ECHO networks to include the number of ECHO sessions delivered within each network and the number attended by each participant. The number of 'low' (attended 1-32\% of sessions), 'medium' (attended 34-67\% of sessions) and 'high attenders' (attended $\geq 68 \%$ of sessions) for each network were calculated. Data were examined and explanations for observed attendance patterns were extracted.

Results The majority of networks delivered nine ECHO sessions held monthly. 1588 participants attended at least once across all 28 networks. Variations in the proportion of low, medium and high attenders were observed across the networks. In $20 / 28$ networks $\geq 50 \%$ of participants were 'low' attenders. Reasons for low attendance included competing workplace demands and staff shortages. However, interview data indicated that for several networks with a high proportion of 'low attenders' participation in the network was at service rather than individual level. Impacts at a service level were still identified through qualitative data collection. Networks with a larger proportion of 'medium' or 'high' attenders were characterised by strong leadership and/or a link to wider service developments.

Conclusion It needs to be considered what outcomes are realistic to assess based on levels of attendance and number of ECHO sessions delivered. Qualitative data collection is recommended to unpick reasons for observed attendance patterns and identify any unexpected outcomes.

\section{P-178 USING THE CONSOLIDATED FRAMEWORK FOR IMPLEMENTATION RESEARCH (CFIR) TO EXPLORE THE USE OF PROJECT ECHO}

${ }^{1}$ Janet Diffin, ${ }^{1}$ Tracey McTernaghan, ${ }^{2}$ Aine McMullan, ${ }^{2}$ Martin Hayes, ${ }^{1}$ Max Watson, ${ }^{1}$ Christopher Jenkins. ${ }^{1}$ Hospice UK, Belfast, UK; ${ }^{2}$ Health and Social Care Board, Belfast, UK
Introduction The Health and Social Care Board identified Project ECHO as a methodology to implement change. The aim was to develop the knowledge and skills of health and social care professionals, transform service delivery, and ultimately improve patient care. Up to 38 ECHO networks across a variety of settings (e.g. palliative care, ophthalmology) were established between 2018-2020.

Aim The aim of this study was to use the Consolidated Framework for Implementation Research (CFIR) to guide assessment of the outcomes and impacts, and explore the factors which influence success across different contexts.

Methods Qualitative study; key stakeholders including high and low attenders, and hub members were purposively invited to participate in a semi-structured interview; interview schedules guided by the CFIR method utilised for data analysis.

Results 28 stakeholders participated. Network purpose varied across contexts, (i) provision of education and support to isolated practitioners; (ii) support practical implementation of strategies/guidelines across sites, (iii) support cross sector working, (iv) build capacity of GPs to manage patients on waiting lists, (v) transform and provide ongoing governance of a new service. Impacts on participants included increased knowledge of conditions and symptoms, increased confidence to treat patients, and increased capacity to manage patients in primary care. Improvements in relationships between primary and secondary care, and across sectors were also reported e.g. improved understanding of regional strategies, service initiatives and referral pathways. Impacts at service level were reported.

Conclusion Project ECHO is an ideal methodology for not only delivering education and support to health and social care professionals, but also as a mechanism for delivering service change. CFIR is a useful framework for identifying preconditions which should be met before a network is operational to ensure success and sustainability.

\section{P-179 A JOINT AGENCY APPROACH TO PROMOTING WELLBEING AND RESILIENCE IN CARE HOME STAFF}

Jane Hazeldine. Mountbatten, Isle of Wight, UK

10.1136/spcare-2021-Hospice. 195

A regular multi-agency meeting 'The Ethical Task Force Group' was created in response to COVID-19 pandemic. It was recognised that staff, managers and owners of care homes were experiencing unique challenges during the pandemic. The task force comprised the Isle of Wight council, the Isle of Wight-Care Partnership and Mountbatten Hospice. The group met weekly to discuss concerns and issues relevant to all parties in order to respond to the needs of care home staff.

One main concern raised was the emotional impact the pandemic was having on care home staff. Many homes lost multiple residents in a short space of time. Often they had known their residents for many years and described their bereavement as the same as losing a family member. They were unable to acknowledge the death in the usual way and therefore were left coping with the ongoing stress of caring for their residents, whilst unable to access their usual support networks and process their bereavement. Both owners and managers of care homes were keen to access strategies and 